\title{
Glucosaminstoffwechsel bei der Aminonucleosid-Nephrose der Ratte ${ }^{1}$.
}

Herrn Professor Dr. H. SARre zum 60. Geburtstag gewidmet

Von W. Reutter und K. Decker

Aus dem Biochemischen Institut der Universität Freiburg i. Breisgaul (Direktor: Prof. Dr. H. Holzer)

(Eingegangen am 24. März 1966)

\begin{abstract}
D-1-14C-Glucosamin wurde normalen und Aminonucleosid-nephrotischen Ratten intravenös verabreicht und der Einbau in die Glycoproteide der Niere und der Leber bestimmt. Es ergaben sich deutliche Unterschiede hinsichtlich der Einbaugeschwindigkeiten und der Umsatzgrößen, die, vor allem in der Niere, vom Stadium der Nephrose abhängig sind. An der in den nephrotischen Ticren etwa verdoppelten Einbaurate in die Nierenproteide ist die glomerulareiche Rindenzone überwiegend beteiligt; dies gilt besonders für die Synthesegeschwindigkeit und die absolute Konzentration der $\mathrm{N}$-Acetylneuraminsäure. Dex Umsatz an proteingebundenem Hcxosamin ist sowohl in der Niere als auch in der Leber Aminonucleosid-behandelter Ratten beträchtlich gesteigert. Quantitative Veränderungen waren ebenfalls in der Markierungsgeschwindigkeit und im Durchsatz nucleotidgebundener Metabolite zu beobachten. Die Befunde werden im Zusammenhang mit morphologischen Beobachtungen und in ihrer Bedeutung für das Verständnis der Nephrose diskutiert.
\end{abstract}

D- $\left[1-{ }^{14} \mathrm{C}\right]$ Glucosamine was administered intravenously to normal and to aminonucleoside-nephrotic rats and its incorporation into the glycoproteins of the kidneys and liver was studied. There were marked differences in the rates of incorporation and rates of turnover, which depended, especially in the kidneys, on the stage of the nephrosis. In the nephrotic animals, the approximately doubled rate of incorporation into kidney proteins is accounted for chicfly by the glomerula-rich cortex; this applies especially to the rate of synthesis and the absolute concentration of $\mathrm{N}$-acetylneuramic acid. The turnover of protein-bound hexosamine is considerably increased in both the kidneys and liver of aminonucleoside-treated rats. Quantitative changes were also observed in the rate of incorporation of label and in the turnover of nucleotide-bound metabolites. These findings, their connection with morphological observations and their significance for the understanding of nephrosis are discussed.

Das Aminonucleosid des Puromycins führt bei der Ratte in spezifischer Weise zur Entwicklung eines Nephrotischen Syndroms, das sich in seinem klinischen und morphologischen Bild von der menschlichen, besonders der kindlichen Lipoidnephrose kaum unterscheidet und deshalb ein wertvolles Versuchsobjekt für das Studium dieser Nierenschädigung darstellt. Deren hervorstechende Merkmale sind: Proteinurie, Hypoproteinämie, Hypercholesterinämie zusammen mit morphologischen Veränderungen, die sich bereits makroskopisch besonders aber elektronenmikroskopisch deutlich manifestieren. Die ersten erkennbaren charakteristischen Veränderungen betreffen die Basalmembranen der Glomerula (1). Diese Strukturen fungieren in der Niere als Filtrierbarriere für hochmolekularè Stoffe (2); ihre Veränderung führt $\mathrm{zu}$ einer Störung dieser Funktion und zu einem Durchlässigwerden für Proteine. Die Basalmembran der Glomerula zeichnet sich durch einen auffallend hohen Gehalt an Hexosamin- und N-Acetylneuraminsäure-haltigen Proteinen aus (3, 3a, 4).

Diese Zusammenhänge veranlaßten uns, den Stoffwechsel der Hexosamine in der Niere näher zu studieren. $\mathrm{Da}$ als Hauptbildungsort für Glycoproteide die Leber angesehen wird $(5,6)$, wurde auch dieses Organ in die Untersuchungen einbezogen. Durch die Bestimmung des Einbaus von markiertem Glucosamin, das eine spezifische und wirksame Vorstufe für die Glycoproteidund die N-Acetylneuraminsäure-Synthese ist, konnten Veränderungen in den Syntheseraten und den Umsatzgrößen verschiedener Metabolite und Glycoproteide in der Aminonucleosid-Nephrose erkannt werden.

1) Mit Unterstützung der Deutschen Forschungsgemeinschaft, Bad Godesberg.

\section{Methodik}

\section{Tierexperimente}

Die Versuche wurden an weiblichen Wistar-Ratten (Durchschnittsgewicht $150 \mathrm{~g}$ ) durchgeführt. Entsprechend der Angabe von FiegelsoN und Mitarbeitern (7) wurde den Tieren während 10 Tagen Aminonucleosid (0,5-proz. in physiologischer Kochsalzlösung) in einer täglichen Dosis von $1,5 \mathrm{mg} / 100 \mathrm{~g}$ subcutan verabreicht. Die Tiere wurden in Stoffwechselkäfigen gehalten; sie erhielten pro Tag je $20 \mathrm{~g}$,Altromin-R“-Standard-Diät und Wasser ad libitum. Im 24-Stdn.-Urin wurde die ausgeschiedene Eiweißmenge mit der Biuretmethode (8) bestimmt. Das Plasmacholesterin wurde nach Richterich und LAUBER (9) ermittelt. Zur Bestimmung der spezifischen Radioaktivität des Serumcholesterins wurden die Proben nach Sperry und Webs (10) aufgearbeitet und das Cholesterin als Digitonid gefällt. Die Serumfettsäuren wurden nach Veresterung mit Diazomethan in ihre Hydroxamsäuren umgewandelt und als solche bestimmt.

Die Versuchstiere wurden in zwei Kollektive eingeteilt: Die Tiere der ersten Gruppe befanden sich in verschiedenen NephroseStadien zwischen dem 6.(beginnende Proteinurie) und 15.Tag (große Proteinurie mit massiver Hypercholesterinämie) nach der ersten Aminonucleosidinjektion. Diese Tiere wurden 3 Stdn. nach der Injektion des markierten Glucosamins getötet. In allen Versuchen wurden $15 \mu C{ }^{1-{ }^{14}} \mathrm{C}-$ Glucosamin in $0,5 \mathrm{ml}$ physiologischer Kochsalzlösung in die Schwanzvene injiziert. Die zweite Gruppe umfaßte Ratten, die sich im gleichen Nephrosestadium (15. Tag nach Beginn der Aminonucleosidbehandlung) mit ausgeprägter Proteinurie befanden. Diese Tiere wurden $z u$ verschiedenen Zeiten (zwischen $5 \mathrm{Min}$. und $20 \mathrm{Stdn}$.) nach der $1{ }^{14} \mathrm{C}-$ Glucosamingabe getötet. Am Ende der Versuchsdauer wurden die Tiere jeweils in leichte Äthernarkose gebracht, Abdomen und Thorax eröffnet, mit einer heparinisierten Rekordspritze möglichst viel Blut durch Herzpunktion gewonnen und dann die Leber, die Nieren und die Milz entnommen. Form, Größe und Aussehen der entnommenen Organe sowie das Vorliegen von Ascites wurden registricrt (Tab. 1). Anschließend wurden die Organe entweder sofort aufgearbeitet oder bei $-15^{\circ}$ aufbewahrt. Bei einem Teil der Tiere wurde nach Eröffnung des Bauchraumes Leber und Niere mittels Gefrierstop nach HoHORst und Mitarbeiter (11) entnommen und dann erst das Herz punktiert. 
Tab. 1. Organgewichte der untersuchten Tiere (Werte in Gramm)

\begin{tabular}{lcccc}
\hline & Normaltiere & $\begin{array}{c}\text { Nephrotische Tiere } \\
(12 .-15 . \text { Tag) }\end{array}$ \\
\hline & Frischgewicht & $\begin{array}{c}\left.\text { Trockengewicht }{ }^{1}\right) \\
\text { in des Frischgewichts }\end{array}$ & $\begin{array}{c}\text { Trockengewicht }{ }^{1} \text { Frischgewicht } \\
\text { in des Frischgewichts }\end{array}$ \\
\hline Leber & $7,05 \pm 0,20$ & $21,1 \pm 2,2$ & $7,95 \pm 0,55$ & $22,7 \pm 4,4$ \\
Nieren & $1,27 \pm 0,14$ & $9,0 \pm 2,9$ & $1,91 \pm 0,31$ & $15,0 \pm 1,0$ \\
Milz & $0,70 \pm 0,18$ & - & $0,41 \pm 0,12$ & - \\
\hline
\end{tabular}

1) Fettfrei

\section{Aufarbeitung der Organe}

Die entnommenen Organe wurden gewogen und in 7-proz. Trichloressigsäure oder in 80-proz. Äthanol im Potter-Elvehjem-Glashomogenisator möglischt vollständig aufgeschlossen und bei Verwendung von Äthanol 1 Std. zum Sieden erhitzt. Das Homogenat wurde bei $2000 \mathrm{~g} 10 \mathrm{Min}$. zentrifugiert, das Sediment so lange mit demselben Fällungsmittel gewaschen, bis keine Radioaktivität mehr im Überstand nachweisbar war. Die Úberstände nach den verschiedenen Zentrifugationen wurden gesammelt und die Radioaktivität gemessen. Die Sedimente wurden mit ChloroformMethanol (2:1) entfettet und in 95-proz. Äthanol aufgeschwemmt.

\section{Alkobol- bz’v. säureunlöslicbe Fraktionen}

Die $N$-Acetylneuraminsäure wurde durch Hydrolyse mit $0,1 \mathrm{~N}$ $\mathrm{H}_{2} \mathrm{SO}_{4}$ bei $80^{\circ}$ (1 Std.) abgespalten (12), der unlösliche Anteil nach dem Abzentrifugieren nochmals der gleichen Prozedur unterworfen. Die Uberstände wurden vereinigt und durch Fraktionierung an Dowex-1 (200-400 mesh, $\left.\mathrm{Cl}^{-}\right)$nach Svennerholm (13) isoliert. Die quantitative Bestimmung erfolgte nach SvENNERHOLM (13) und Warren (14) in der Modifikation von Feuerstein und GEYER (3).

Zur Isolierung des proteingebundenen Glucosamins wurden die Proben unter Rückfluß in $4 \mathrm{~N}_{2} \mathrm{SO}_{4} 3 \mathrm{Stdn}$. bei $100^{\circ}$ hydrolysiert, die Schwefelsäure mit gesättigter Barytlauge neutralisiert und der Hexosamingehalt in einem Aliquot bestimmt (15).

\section{Alkobollösliche Fraktionen}

Die Gewinnung der markierten Nucleotidfraktionen geschah in Anlehnung an die Methode von O'Brien und Mitarbeiter (16): Der äthanollösliche Überstand wurde nach Entfernung des Äthanols mit Chloroform-Methanol (2:1) entfettet, mit Essigsäure angesäuert, die Nucleotide an aktivierte Tierkohle adsorbiert, das Kohlesediment nach dem Abzentrifugieren mit 0,01 N Essigsäure gewaschen, und der Vorgang so lange wiederholt, bis keine Radioaktivität mehr in der Waschflüssigkeit nachweisbar war. Die Überstände wurden gesammelt. Die Nucleotide wurden von der Kohle mit ammoniakalischem Äthanol (1 ml 25-proz. Ammoniaklösung auf 1 l 50-proz. Äthanol) vollständig eluiert. Die vereinigten Nucleotidfraktionen wurden zur Entfernung des Äthanols am Rotationsverdampfer eingeengt, nochmals bei $10000 \mathrm{~g}$ zentrifugiert, um restliche Kohlepartikel zu entfernen, und der Rest chromatographisch aufgetrennt.

\section{Chromatographie}

Für die N-Acetylneuraminsäure eignet sich n-Butanol/n-Propanol/ $0,1 \mathrm{~N}$ Salzsäure (1:2:1) (17) und Pyridin/Essigester/Essigsäure/ Wasser (5:5:1:3) (18) auf MN-Zellulose 300 G-Dünnschichtplatten. Letzteres wurde, neben Pyridin/Butanol/Wasser $(6: 4: 3)$ (19), auch zur Auftrennung der Aminozucker und deren NAcetylderivate verwandt. Die weitere Identifizierung erfolgte durch Hochspannungselektrophorese $\left(2^{1} / 2 \mathrm{Stdn} ., 0^{\circ}, 18 \mathrm{~V} / \mathrm{cm}\right)$ in 1-proz. Kaliumtetraboratpuffer $\left(\mathrm{K}_{2} \mathrm{~B}_{4} \mathrm{O}_{7} \cdot 4 \mathrm{H}_{2} \mathrm{O}\right) \mathrm{pH} 8-9$ (20). Die Auftrennung des Nucleotidgemisches erfolgte auf Whatman3-Papier im Fließmittel Äthanol/1MAmmoniumacetat (15:6) pH 7,6 und Rechromatographie im gleichen Fließmittel bei pH 3,8 (21). Zur weiteren Reinigung und Identifizierung der einzelnen markierten Nucleotidfraktionen dienten die von CARMINATTI und Mitarbeitern (22) angegebenen borathaltigen Fließmittel, die Glucosamin-, Mannosamin- und Galactosamin-haltige Nucleotide zu trennen vermögen: 1. Åthanol/Methyläthylketon/0,5 м Morpholintetraborat $\mathrm{pH} 8,6(7: 2: 3) ; 2$. Dimethoxyäthan/Methyläthylketon/0,5 м Morpholintetraborat $\mathrm{pH} 8,6$ (7:2:3); ferner Âthoxyäthanol/Butanol/Wasser $(4: 1: 1)$.

Der Aminozucker- und N-Acetylneuraminsäure-Nachweis erfolgte allgemein mit Silbernitrat + Alkali nach Trevelyan und Mitarbeiter (23); speziell auch mit Ninhydrin (für Aminozucker), dem Morgan-Elson-Reagenz in einer Modifikation von Russe und LÜDERITz (24) (für N-Acetyl-Aminozucker) und nach AMINOFF (25) (für N-Acetylneuraminsäure).

\section{Radioaktivitätsmessungen}

Die Bestimmung der Radioaktivität in den einzelnen Proben erfolgte in einem Szintillationsspektrometer (Tri-carb 314 X, Fa. Packard Instrument Company). Zur Messung des ${ }^{14} \mathrm{C}$-Einbaues in die Proteinfraktion ließen wir die größeren Partikel der Äthanolaufschwemmung 5 Stdn. sedimentieren und brachten mittels Saugfiltration ein Aliquot der feinsten Partikelsuspension auf ein Filtrierpapier (Schleicher \& Schüll Nr. 597) von 2,5 cm Durchmesser. Nach der Trocknung wurde die Probe auf einer Mikroanalysenwaage gewogen. Der Proportionalitätsbereich lag zwischen 0,8 und $4 \mathrm{mg}$ Proteinniederschlag pro Blättchen. Die aufgetragene Menge betrug im Mittel 1,5 mg.

\section{Materialien}

Das Aminonucleosid des Puromycins 6-(Dimethyl-amino-)9-(3'desoxy-3'-amino-D-ribosyl)-purin wurde bezogen von der $\mathrm{Fa}$. „Nutritional Biochemicals Corporation“, Cleveland, Ohio, USA; ${ }_{1-14} \mathrm{C}-\mathrm{D}-$ Glucosamin-Hydrochlorid von $\operatorname{der} \mathrm{Fa}$. „New England Nuclear Corporation“", Boston, USA; 2-14C-Uridin von „The Radiochemical Centre", Amersham, England; D-Glucosamin . $\mathrm{HCl}$, A grade, von der Fa. „Calbiochem“, Los Angeles, USA; $\mathrm{D}$-Galactosamin $\cdot \mathrm{HCl}, \mathrm{D}-\mathrm{Mannosamin} \cdot \mathrm{HCl}, \mathrm{N}$-Acetyl-D-Glucosamin, N-Acetyl-Galactosamin und $\mathrm{N}$-Acetylneuraminsäure von der Fa. „Sigma Chemical Company“, St. Louis, USA; N-AcetylD-Mannosamin von der Fa. „Koch-Light Laboratories Ltd.“, Colnbrook, England. UDP-N-Acetylglucosamin wurde uns von der Fa. Boehringer, Mannheim, in dankenswerter Weise überlassen.

\section{Ergebnisse}

\section{Zeitabbängigkeit der Glucosamininkorporation}

In ersten, informativen Versuchen verglichen wir den Einbau von markiertem Glucosamin in den säureunlöslichen Anteil der gesamten Niere normaler und Aminonucleosid-nephrotischer Ratten und fanden ihn in den nephrotischen Tieren 3 Stdn. nach Injektion der markierten Verbindung auf das etwa Zweifache erhöht.

Mehr Aufschluß über die Unterschiede zwischen nephrotischen und normalen Tieren erhielten wir jedoch bei der Beobachtung des zeitlichen Ablaufs der Glucosamininkorporation in Leber und Niere (Abb. 1). Die Einbeziehung der Leber läßt erkennen, daß nach Aminonucleosidgabe auch in diesem Organ ein veränderter Hexosaminstoffwechsel besteht. Aus dem Vèr- 


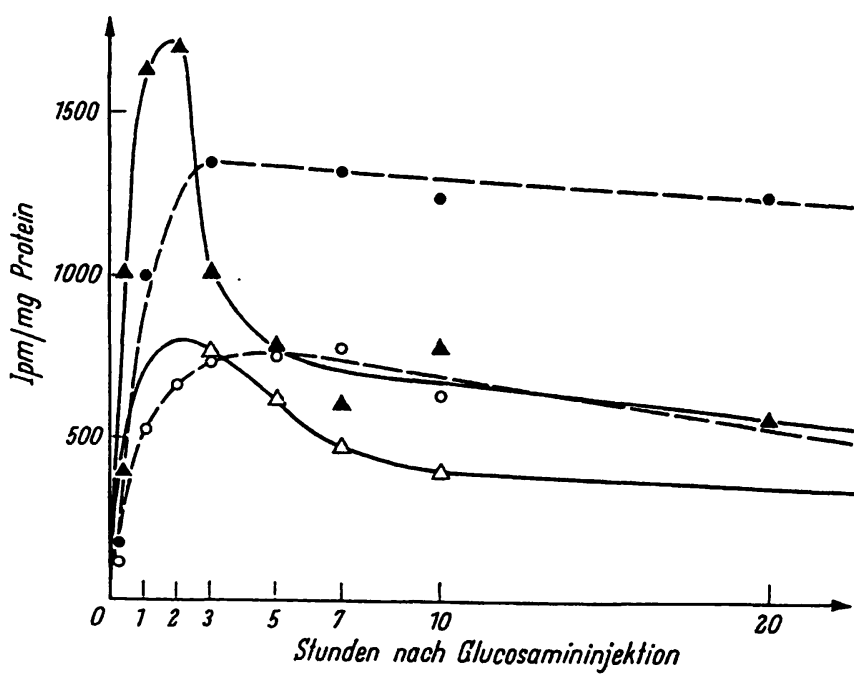

Abb. 1

${ }^{1-1.4} \mathrm{C}-$ Glucosamineinbau in die säureunlösliche Fraktion von Niere und Leber

$$
\begin{aligned}
\text { Niere }--0 & =\text { normal } & \text { Leber }-\Delta & =\text { normal } \\
0 & =\text { nephrotisch } & & =\text { nephrotisch }
\end{aligned}
$$

lauf der Kurven ist zu ersehen, daß der Umsatz des fixierten Hexosamins nicht nur in der Nephrose, sondern auch normalerweise in der Leber größer ist als in der Niere; in der nephrotischen Leber ist er gegenüber der Norm nochmals beträchtlich gesteigert. Man findet hier auch das Maximum des Glucosàmineinbaus bereits nach 2 Stdn., während es in der Niere erst nach etwa 3 Stdn. erreicht wird.

Die säureunlöslichen Glycoproteide wurden durch fraktionierte Hydrolyse weiter unterteilt, wobei man durch Behandlung mit $0,1 \mathrm{~N} \mathrm{H}_{2} \mathrm{SO}_{4}\left(80^{\circ}, 1 \mathrm{Std}\right.$.) als markiertes Hydrolysenprodukt fast ausschließlich $\mathrm{N}$ Acetylneuraminsäure erhält, während nach Einwirkung von $4 \mathrm{~N} \mathrm{H}_{2} \mathrm{SO}_{4}\left(100^{\circ}, 3 \mathrm{Stdn}\right.$.) die abgespaltene Radioaktivität überwiegend als Glucosamin vorliegt. Das Ergebnis dieser Versuchsreihe (Abb. 2) zeigt, daß sich in den Nieren der nephrotischen Tiere die N-Acetylneuraminsäure-reiche Fraktion schnell markiert, aber metabolisch stabil ist, während die Glucosamin (bzw. NAcetyl-Glucosamin) enthaltenden Glycoproteide einem. raschen Umsatz unterliegen.

Aus der Darstellung in Abbildung 3 läßt sich entnehmen, daß an der Steigerung der Gesamtinkorporation des Glucosamins die erhöhte Synthese von N-Acetylneuraminsäure prozentual am meisten beteiligt ist. Ein besonderes Gewicht erbält dieser Befund durch die weitere Beobachtung, daß man bei einer Aufteilung der Niere in eine glomerulareiche Rinden- und eine Markzone die Zunahme des ${ }^{14} \mathrm{C}-$ Gehalts vor allem auf den Rindenbereich konzentriert findet (Tab. 2). In der Cyclohexanonphase des Warren-Tests wurde nicht nur

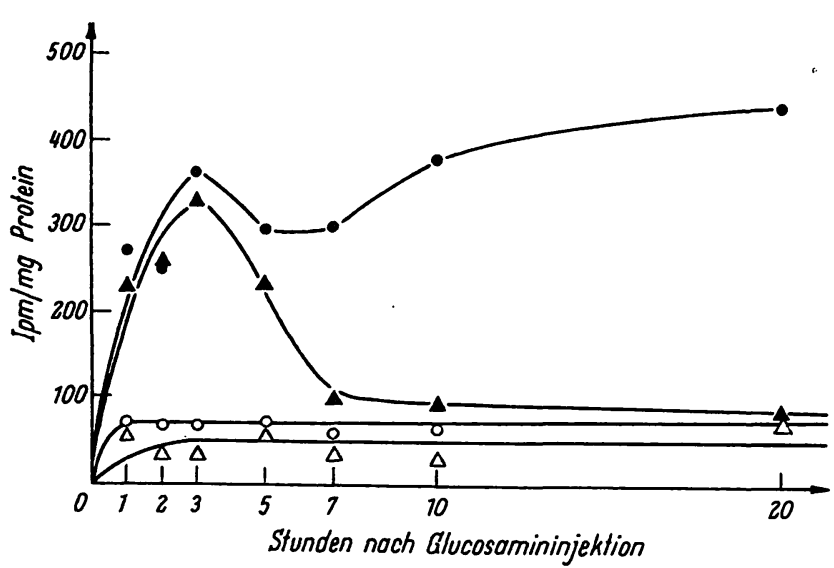

Abb. 2

Zeitlicher Verlauf des ${ }^{14} \mathrm{C}$-Einbaues in verschiedene Glycoproteidfraktionen der Niere nach 1-1.1. C-Glucosamingabe

Von einer bekannten Menge an TES-unlöslichem Proteinniederschlag wurde in Lösung gebracht a) durch Hydrolyse in $0,1 \mathrm{~N}$ $\mathrm{H}_{2} \mathrm{SO}_{4}$ (1 Std., $80^{\circ}$ ) O-O Normalniere, 0-O nephrotische Niere; b) durch weitere Hydrolyse in $4 \mathrm{~N} \mathrm{H}_{2} \mathrm{SO}_{4}$ (3 Stdn., 100 ) $\Delta-\Delta$ Normalniere, $\Delta-\Delta$ nephrotische Niere

die quantitative kolorimetrische Bestimmung durchgeführt; die dort aufgefundene Radioaktivität ist bei Verwendung von $1-{ }^{14} \mathrm{C}$-Glucosamin ein spezifisches $\mathrm{Maß}$ für die Inkorporation in die Neuraminsäure.

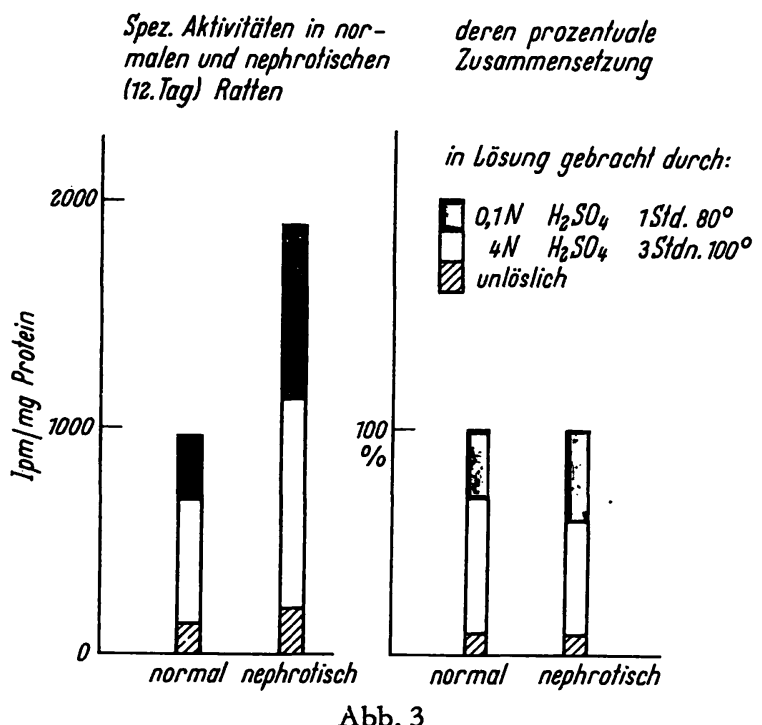

Relative Verteilung der Radioaktivität in verschiedenen Glycoproteidfraktionen der Niere 4 Stdn. nach ${ }^{1-14} C$-Glucosamininjektion

\section{Abbängigkeit vom Nephrosestadium}

Da unser besonderes Interesse denjenigen biochemischen Vorgängen galt, die in ursächlichem Zusammenhang mit der Entstehung des Nephrotischen Syndroms stehen, studierten wir das Eintreten des erhöhten Hexosamineinbaus in Abhängigkeit vom Nephrosestadium (Abb. 4). Man sieht, daß in der Niere, im Gegensatz zur Leber, die

Tab. 2. Gebalt und spezifische Radioaktivität der N-Acetylneuraminsäure im Trichloressigsäure-Unlöslichen nach ${ }^{1-14} \mathrm{C}-\mathrm{Glucosamin}$ injektion. Die Rinde wurde vom Mark als eine etwa $1 \mathrm{~mm}$ dicke Schicht abgetrennt und beide gesondert aufgearbeitet

\begin{tabular}{lccc}
\hline & \multicolumn{2}{c}{ Kontroll-Nieren } & \multicolumn{2}{c}{$\begin{array}{c}\text { Nephrot. Nieren } \\
\text { Rinde }\end{array}$} & Mark & Rinde & 2,91 \\
nMol N-Acetylneuraminsäure/mg TES-Unlösliches & 1,88 & 0,95 & 225 \\
IpM/nMol N-Acetylneuraminsäure & 105 & 1,52 & 127 \\
\hline
\end{tabular}




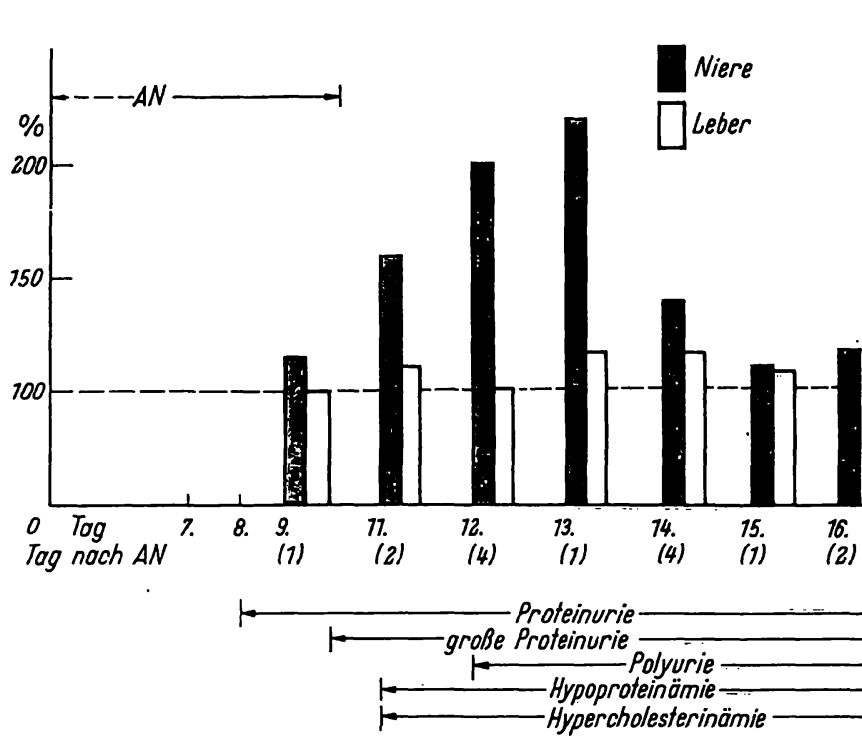

Abb. 4

Einbau von $1{ }^{14} \mathrm{C}$-Glucosamin in Leber und Niere in Abhängigkeit vom Nephrosestadium

Angaben in Prozent der Normaltiere. Niere, $\square$ Leber

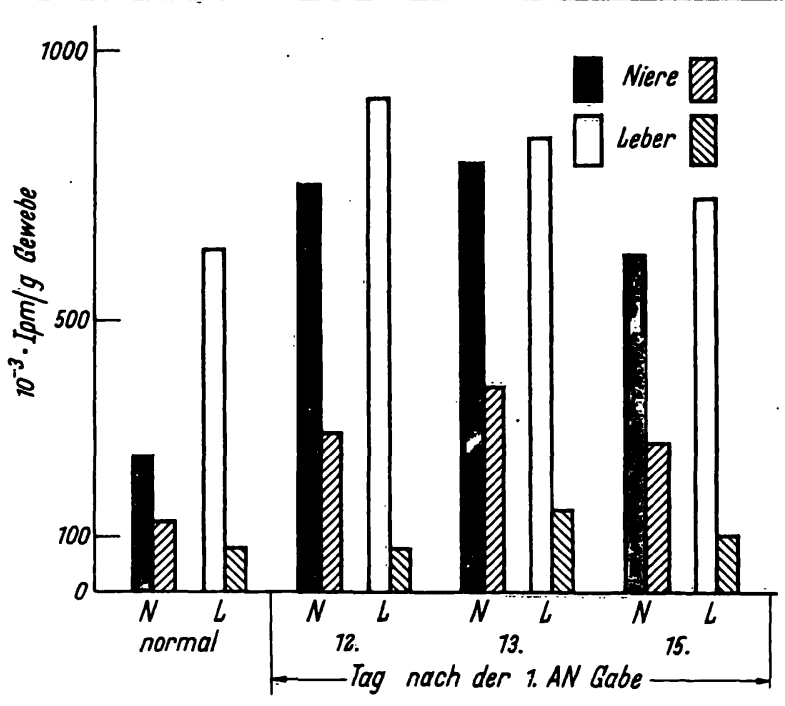

Abb. 5

${ }^{1-14} \mathrm{C}-$ Glucosamineinbau in den äthanollöslichen Überstand von Nieren und Lebern in Abhängigkeit vom Nephrosestadium.

15 Min. nach Glucosamingabe (Leber $\square$, Niere

4 Stdn. nach Glucosamingabe (Leber $\mathbb{N}$, Niere $\% / /$ )

Paladini-Fließmittel zeigte sich eine stark markierte Zone (Abb. $6 \mathrm{a} ; R_{\mathrm{F}}=0,45$ ), die sich als ein Gemisch von UPD-N-Acetyl-glucosamin und =galactosamin identifizieren ließ. Diese Fraktion erwies sich bei den nephrotischen Tieren sowohl in der Niere als auch in der Leber stets höher markiert als in den Kontrolltieren. Das Maximum der spezifischen Aktivität wird in den gesunden Tieren nach 15 Min. erreicht (vgl. dazu (6)); in den nephrotischen ist es nach 33 Min. noch nicht überschritten. Wenig verändert war dagegen in beiden Tiergruppen das Verhältnis beider Substanzen, dạs 5 Min. nach Injektion des markierten Glucosamins 3:1 zugunsten des UDP-N-Acetyl-glucosamins betrug, nach $30 \mathrm{Min}$. jedoch eine konstante Relation von 2:1 erreichte. Die metabolische Labilität dieser Verbindungen zeigte sich bèi einem Vergleich von friergestoppten mit konventionell entnommenen Lebern: die UDP-N-Acetyl-glucosamin- und -galactosamin-Werte fanden wir nach Frierstop durchweg um etwa $20 \%$ höher.

Neben diesen beiden bekannten UDP-N-Acetylaminozuckern trat im Paladini-System eine schneller $\left(R_{F}=0,70-0,78\right)$ wandernde, markierte Zone auf, die durch Rechromatographie im gleichen Fließmittel (bei längerer Laufzeit), besser aber in Äthoxyäthanol/Butanol $/ \mathrm{H}_{2} \mathrm{O}(4: 1: 1)$, in $z$ wei Komponenten ( $\beta$ und $\gamma$ der Abb. 6) aufzutrennen war. $\gamma$ nahm mit fortschreitender Versuchsdauer gegenüber $\beta$ zu ( $\beta: \gamma$ nach 5 Min.: 15:1; nach 22 Min.: 1:1; nach 33 Min.: 1:2). Betrachtet man den prozentualen Anteil von $\beta+\gamma$ an der Gesamtmarkierung der nucleotidgebundenen Metabolite, so findet man ihn in den nephrotischen Tieren, besonders in der

Tab. 3. Auftreten von Radioaktivität im Serumcholesterin nach $1-{ }^{14} \mathrm{C}-$ Glucosamininjektion

\begin{tabular}{lcccccc}
\hline & & & \multicolumn{2}{c}{ Nephrotische Tiere } & \multicolumn{2}{c}{ Kontrolltiere } \\
\hline Min. nach 1-14 C-Glucosamingabe & 16,5 & 18 & 27 & 27 & 15 & 25 \\
IpM/ $\mu$ Mol Cholesterin & 4,3 & 8,5 & 23,3 & 54 & 4,1 & $6,5 \ldots$ \\
\hline
\end{tabular}



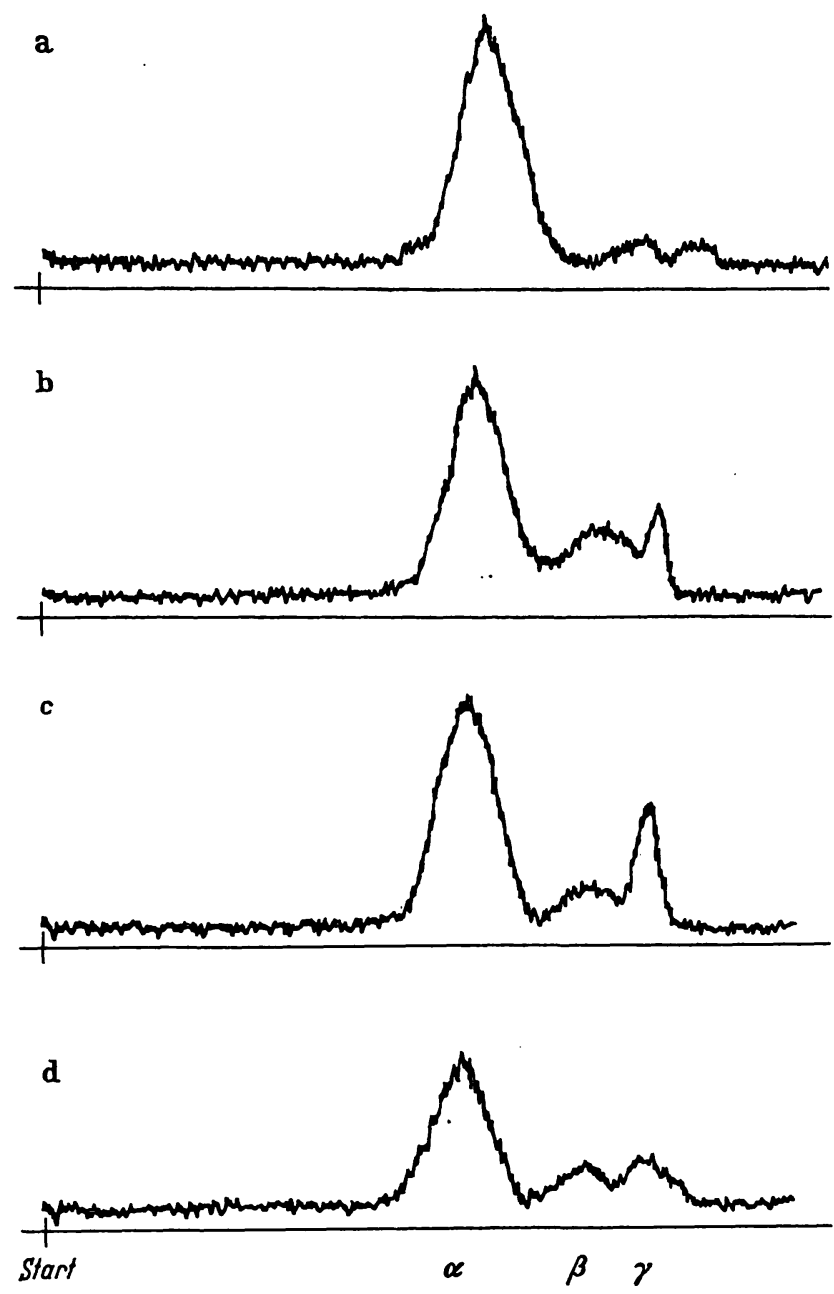

Abb. 6

Chromatographische Trennung der nucleotidgebundenen Hexosamine aus nephrotischen Rattenlebern

Zeitpunkt der Organentnahme nach Gabe von $1{ }^{14} \mathrm{C}-\mathrm{Glucosamin}$ $(2,78 \mu \mathrm{C} / \mu \mathrm{Mol})$ :

a) 15 Min. (Frierstop), b) $15 \mathrm{Min}$. (ohne Frierstop), c) $33 \mathrm{Min}$. (ohne Frierstop)

d) $15 \mathrm{Min}$. nach Gabe von $2-{ }^{14} \mathrm{C}$-Utridin $(36,9 \mu \mathrm{C} / \mu \mathrm{Mol})$ (Frierstop). Flie/smittel: 1MAmmoniumacetat/Äthanol (15:6), pH 7,6 (18 Stdn., Whatman 3)

Leber (Tab. 4), erhöht. Auch bei konventioneller Aufarbeitung erhielten wir - sowohl mit Lebern normaler als auch nephrotischer Tiere - im Vergleich zum Frierstop durchweg höhere Werte.

\section{Diskussion}

Die dargestellten Ergebnisse vermitteln das Bild eines in der Aminonucleosid-nephrose beträchtlich gesteigerten
Hexosaminstoffwechsels in Leber und Niere, der sich durch erhöhte absolute und spezifische Aktivitäten des inkorporierten ${ }^{1-{ }^{14}} \mathrm{C}$-Glucosamins manifestiert. Die größere Synthesegeschwindigkeit und vor allem Umsatzrate der Glycoproteide der Leber hängt in erster Linie mit dem durch die Proteinurie bedingten Mehrbedarf an Plasmaglycoproteiden zusammen; die Herkunft dieser Eiweißfraktion des Plasmas aus der Leber ist mehrfach nachgewiesen worden $(26,27)$, sehr eindeutig z. B. von SHETLAR (28) durch Darstellung einer kinetischen Vorläufer-Produkt-Beziehung („ZilversmitKurven" (29)). Aus dem zeitlichen Verlauf der spezifischen Aktivität der Glycoproteidfraktion von Leber und Niere (Abb. 1) kann man auf eine ähnliche Beziehung zwischen diesen beiden Organen schließen, die durch das Blutplasma vermittelt wird. Sie ist nicht nur im Hinblick auf die spezielle Fragestellung der Nephrose, sondern ganz allgemein von Bedeutung; sie würde besagen, daß wenigstens ein Teil der Glycoproteide der Niere nicht in diesem Organ selbst, sondern in der Leber gebildet und in die Nierenzellen eingebaut wird.

Eine funktionell und metabolisch ausgezeichnete Rolle unter den Glycoproteiden kommt den sog. Sialoproteiden zu, welche N-Acylneuraminsäuren enthalten. Es ist bekannt, daß diese Verbindungen wichtige Bestandteile von Zellmembranen sind und ihre Entfernung durch Neuraminidase $z u$ drastischen Veränderungen im $\mathrm{La}_{-}$ dungsmuster (Mobilität im elektrischen Feld (30-32)) und in den Permeabilitätseigenschaften (33) führt. Der ausnehmend hohe Gehalt an Sialinsäure in den für die Filtrationsleistung der Glomerula bedeutungsvollen Basalmembranen $(3,3 a, 4)$ legt einen Zusammenhang $z$ wischen gestörter Filtrationsfunktion der Niere und den Sialoproteiden der glomerulären Membranstrukturen nahe (34). Die ersten morphologisch erkennbaren Veränderungen in der Aminonucleosid-nephrose bestehen in einer Verformung und Verdickung dieser Basalmembranen. Wir legen deshalb dem Befund, daß Veränderungen in der Syntheserate von Glycoproteiden der Niere bei dieser Erkrankung die ersten nachgewiesenen biochemischen Veränderungen darstellen und bereits zum Zeitpunkt des ersten Auftretens einer Proteinurie erkennbar sind, besondere Bedeutung bei. Die in der Abbildung 4 dargestellten Versuchsdaten zeigen darüber hinaus, daß die starke Erhöhung der Syntheseraten in der Niere nur bis zu einem Zeitpunkt anhält, bei dem auch das Maximum der Proteinurie erreicht ist, um dann all-

Tab. 4. Prozentualer Anteil der Komponenten $\beta$ und $\gamma$ an der Gesamtmarkierung der Nucleotidfraktion Die Zahlen stellen den Quotienten $\frac{\beta+\gamma}{\alpha+\beta+\gamma} \cdot 100 \mathrm{dar}$

\begin{tabular}{|c|c|c|c|c|}
\hline \multirow{2}{*}{$\begin{array}{l}\text { Min. nach } \\
{ }^{14} \mathrm{C} \text {-Glucosamingabe }\end{array}$} & \multicolumn{2}{|c|}{$\begin{array}{c}\text { Kontrolltiere } \\
\text { Frierstop }\end{array}$} & \multicolumn{2}{|c|}{$\begin{array}{c}\text { Nephrotische Tiere } \\
\text { Frierstop }\end{array}$} \\
\hline & mit & ohne & mit & ohne \\
\hline 15 & 5,8 & 10,2 & 10,6 & $\begin{array}{l}13,0 \\
20,0\end{array}$ \\
\hline 22 & 6,3 & 13,0 & $\begin{array}{l}7,6 \\
8,6\end{array}$ & $\begin{array}{l}27,8 \\
15,9\end{array}$ \\
\hline 33 & - & - & 11,4 & $\begin{array}{l}33,4 \\
13,6\end{array}$ \\
\hline
\end{tabular}


mählich wieder abzusinken. Eine ganz analoge Verlaufsform hatten wir hinsichtlich der Cholesterinsynthese in der Aminonucleosid-nephrose gefunden (35); auch hier wurde zunächst durch eine stark gesteigerte Bildungsgeschwindigkeit ein erhöhter Cholesterinspiegel des Plasmas erzeugt, der dann durch eine nahezu normalisierte Syntheserate aufrechterhalten werden konnte. Die Tabelle 2 beweist, daß auch die Sialoproteide der Niere vermehrt gebildet werden und in erhöhter Konzentration vorliegen; im Gegensatz zu den anderen Glycoproteiden ist der Umsatz der vorwiegend aus Sialoproteiden bestehenden Fraktion (Abb. 1) gering. Dabei ist es besonders bemerkenswert, daß innerhalb der Niere die glomerulareiche Rindenzone sowohl hinsichtlich der Syntheseleistung als auch des N-Acetylneuraminsäuregehalts eine bevorzugte Stellung einnimmt. Diese biochemischen Befunde stehen mit den morphologischen Beobachtungen an Aminonucleosid-nephrotischen Rattennieren in guter Übereinstimmung.

Eine Folge der gesteigerten Bildungsgeschwindigkeiten verschiedener Glycoproteide ist der größere Umsatz an Zwischenprodukten dieser Synthesen. Während für die vorwiegend $\mathrm{N}$-Acetyl-glucosamin als Aminozuckerkomponente enthaltenden Glycoproteide ein unmittelbarer Zusammenhang mit der schnelleren Markierung des UDP-N-Acetylglucosamins besteht, verläurt die Synthese der N-Acylneuraminsäuren über weitere, bislang noch nicht vollständig aufgeklärte Zwischenstufen. Wir sind in diesem Zusammenhang an den in Abbildung 6 dargestellten, während der Nephrose vermehrt auftretenden Verbindungen besonders interessiert, da sie möglicherweise noch unbekannte Zwischenstufen der N-Acetylneuraminsäuresynthese darstellen. Eine Úbersicht über diejenigen biochemischen Prozesse, die mit dem Auftreten eines Nephrotischen Syndroms nach Aminonucleosidbehandlung quantitative oder qualitative Änderung erfahren, wird in dem folgenden Schema versucht. Es muß noch dahingestellt bleiben, ob es sich bei dem vermehrten Einbau von Sialo- und Glycoproteiden in die Niere bereits um eine Reparaturleistung des Organismus zur Beseitigung der durch das Aminonucleosid ausgelösten Schädigung handelt. Umgekehrt könnte die Wirkung des Aminonucleosids auch in einer Störung der Regulation der Membranbildung bestehen und so einen verstärkten Eintritt der Glycoproteide veranlassen.

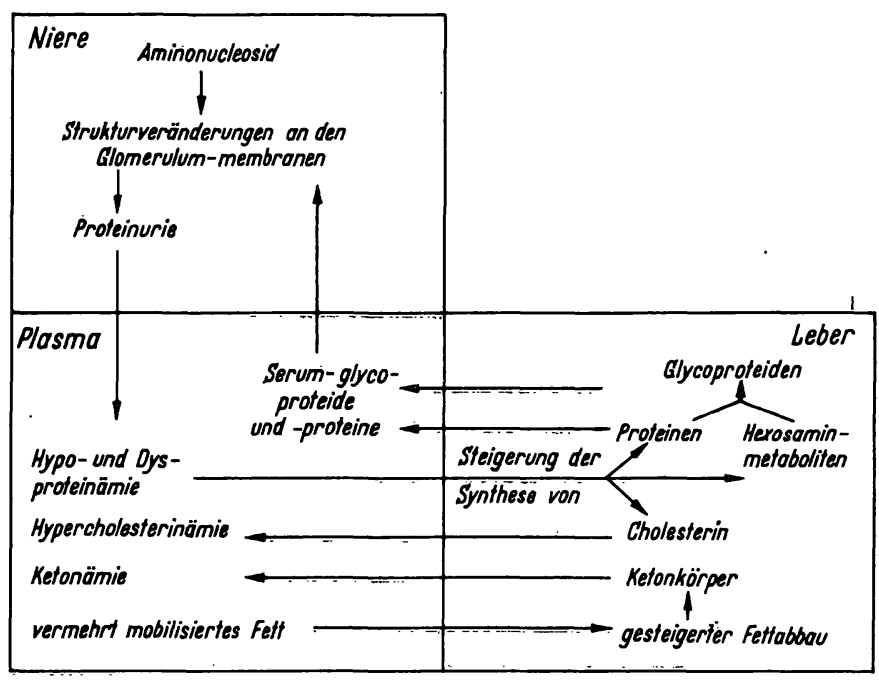

\section{Literatur}

1. DubaCh, U. C., Aminonucleosidnephrose, in Fortschritte der Arzneimittelforschung, hrsg. von E. Jucker, Bd. VII, S. 341. Birkhäuser Verlag, Basel-Stuttgart (1964). - 2. FARQuHAR, M. G. und G. E. Palade J. Exper. Med. 114, 699 (1961). - 3. Feuerstern, H. und G. GeYer, Acta biol. med. german. 11, 1 (1963). 3a. GeYer, G., Naturwissenschaften 49, 183 (1962). - 4. LANGE, C. F. und A.S. Markowrtz, Biochim. biophysica Acta (Amsterdam) 101, 217 (1965). - 5. Winzler, R., The Amino Sugars, hrsg. von E. A. Balazs und R. W. Jeanloz, Bd.IIA, S. 338, Academic Press, New York-London (1965). - 6. MACBETH, R. A. L., J. G. BekESI, E. Sudgen und S. BrCE, J. biol. Chemistry 240, 3707 (1965). - 7. Fiegelson, E. B., J. W. Drake und L. Recaut, J. Lab. clin. Med. 50, 437 (1957). - 8. Beisenherz, G., H. J. Boltze, Th. Bücher, R. Czok, K. H. Garbade, E. Meyer-Arendt und G. Pfleiderer, Z. Naturforsch. 86, 555 (1953). - 9. RichteRICH, R. und K. LaUber, Klin. Wschr. 40, 1252 (1962). - 10. SpERRY, W. M. und M. WeBb, J. biol. Chemistry 187, 97 (1950). — 11. HohoRst, H. J., F. H. KREUTZ und Th. Bücher, Biochem. Z. 332, 18 (1959). 12. Gotrschalk, A., Biochim. biophysica Acta (Amsterdam) 20, 560 (1956). - 13. Svennerholm, L., Methods in Enzymology, Bd. VI, S. 459 Academic Press inc, New York-London (1963). 14. Warren, L., J. biol. Chemistry 234, $1971 \cdot(1959)$. - 15. Boas, N. F., J. biol. Chemistry 204, 553 (1953). - 16. O'Brren, P. J. und E. Neufeld, Biochim. biophysica Acta (Amsterdam) 83, 352 (1964). - 17. Svennerholm, E. und L. Svennerholm, Nature
(London) 181, 1154 (1958). - 18. Fischer, F. G. und H. J. NEBEL, Hoppe-Seyler's Z.f.physiol. Chem.302, 10 (1955). - 19. CRUMPton,; M. J., Biochem. J. 72, 479 (1959). - 20. Maley, F. und G. F. MALEY, Biochim. biophysica Acta (Amsterdam) 31, 577 (1959). 21. Palndini, A. C. und L. F. Leloir, Biochem. J. 51, 426 (1952). - 22. Carminatti, H., S. Passeron, M. Dankert und E. ReconDo, J. Chromatogr. (Amsterdam) 18, 342 (1965). - 23. Trevelyan, W. E., D. P. Procter und J. S. Harrison, Nature (London) 166, 444 (1950). — 24. Rrsse, H. J. und O. Lüderitz, Biochem. Z. 341, 1 (1964). - 25. Aminoff, D., Biochem. J. 81, 384 (1961). 26. Robinson, G. B., J. Molnar und R. H. Winzler, J. biol. Chemistry 239, 1134 (1964). - 27. Rrchmond, J. E., Biochemistry 2, 676 (1963). - 28. Shetrar, M. R., J. C. Capps und D. L. Hern, Biochem. biophysica Acta (Amsterdam) 83, 93 (1964). - 29. Zilviersmít, D. B., C. Entenman und M. C. Fischler, J. gen. Physiol. 26, 325 (1948). - 30. Langlex, O. K., und E. J. Ambrose, Nature (London) 204, 53 (1964). - 31. RuhenstrothBauer, G., W. Kübler, G. F. Fuhrmann und F. RuefF, Klin. Wschr. 39, 764 (1961). - 32. Forrester, J. A. E. J. AMbrose und M. G. P. Stoker, Nature (London) 201, 945 (1964). 33. Glick, J. L. und S. Githens III, Nature (London) 208 , 88 (1965). - 34. v. Bruchhausen, F. und H. J. Merker, NaunynSchmiedebergs Arch. exp. Pathol. Pharmakol. 251, 1 (1965). - 35. Kattermann, R., W. Reutter und K. Decker, Klin. Wschr. 43, 393 (1965).

Doz. Dr. K. Decker

Biochemisches Institut der Universität 78 Freiburg/Br. Hermann-Herder-Str̀. 7 